\title{
Biological potential of apigetrin in the medicine for the treatment of various forms of inflammatory disorders: Phytotherapeutic approach for respiratory infections
}

\author{
Dinesh Kumar PATEL*
}

Department of Pharmaceutical Sciences, SHUATS, Prayagraj, India

Introduction: Apigetrin is apigenin-7-O-glucoside is a natural flavonoid glucoside also called flavonoidal class plant derive phytochemical found to be present in the nature in Matricaria chamomilla, Scutellaria baicalensis, Stachys tibetica and Teucrium gnaphalodes.

Methods: In order to know the medicinal potential of apigetrin in the medicine and health sectors, here numerous scientific data have been collected from various literature sources and analyzed for their health beneficial potential. Medicinal importance and pharmacological activities data of apigetrin have been collected from various literature sources and analyzed for their therapeutic potential in the medicine. Molecular mechanism of apigetrin in different disorders data have been also collected from various literature sources and analyzed for their cellular action. All the collected scientific data have been properly analyzed to get the better result in the present investigation.

Results: Scientific data of various literature sources revealed the biological potential of apigetrin in the medicine for the treatment of numerous health complications. From the data analysis of different scientific work of the literature sources it was found that apigetrin have neuroprotective potential due to their anti-inflammatory activity and inhibitory potential on oxidative stresses. Further biological effect on lipopolysaccharides (LPS)-induced otitis media showed its anti-inflammatory and anti-oxidant potential. Literature data analysis signified the biological role of TNF- $\alpha$ for their anti-inflammatory activity. Literature database analysis also revealed their biological importance to treat inflammatory diseases of upper respiratory infections.

Conclusions: Scientific data analysis of various literature works revealed the biological potential of apigetrin in the medicine for the treatment of various form of inflammatory disorders. 